Research Paper

\title{
The Role of Adjuvant Chemotherapy for Colorectal Liver Metastasectomy after Pre-Operative Chemotherapy: Is the Treatment Worthwhile?
}

\author{
Yun Wang1,2*, Zhi-qiang Wang1,2*, Feng-hua Wang1,2, Yun-fei Yuan¹,3, Bin-kui Li1,3, Pei-rong Ding1,4, \\ Gong Chen ${ }^{1,4}$, Xiao-jun Wu'1,4, Zhen-hai Lu1,4, Zhi-zhong Pan ${ }^{1}{ }^{1}$, De-sen Wan ${ }^{1,4}$, Peng Sun ${ }^{1,5}$, Shu-mei Yan ${ }^{1}$

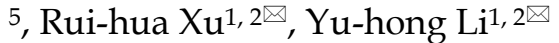 \\ 1. Sate key Laboratory of Oncology in South China, Sun Yat-sen University Cancer Center, Collaborative Innovation Center for Cancer Medicine, Guangzhou, \\ P.R. China; \\ 2. Department of Medical Oncology, Sun Yat-sen University Cancer Center, Guangzhou, P.R. China; \\ 3. Department of hepatobiliary surgery, Sun Yat-sen University Cancer Center, Guangzhou, P.R. China; \\ 4. Department of colorectal surgery, Sun Yat-sen University Cancer Center, Guangzhou, P.R. China; \\ 5. Department of Pathology, Sun Yat-sen University Cancer Center, Guangzhou, P.R. China. \\ *These authors contributed equally to this work
}

$\square$ Corresponding authors: Professor Yu-Hong Li and Rui-hua Xu, Sate key Laboratory of Oncology in South China and Department of medical oncology, Sun Yat-sen University Cancer Center, Collaborative Innovation Center for Cancer Medicine, P.R. China; E-mail: liyh@sysucc.org.cn; xurh@ sysucc.org.cn.

(c) Ivyspring International Publisher. This is an open access article distributed under the terms of the Creative Commons Attribution (CC BY-NC) license (https://creativecommons.org/licenses/by-nc/4.0/). See http://ivyspring.com/terms for full terms and conditions.

Received: 2016.10.26; Accepted: 2017.01.12; Published: 2017.04.09

\begin{abstract}
Peri-operative chemotherapy has been proposed to improve the survival of patients with colorectal cancer hepatic metastases (CRCHM). However, the role of the adjuvant chemotherapy post-metastasectomy for CRCHM patients who have undergone pre-operative chemotherapy is still undetermined. We retrospectively analyzed the role of adjuvant chemotherapy post-metastasectomy on relapse-free survival (RFS) and overall survival (OS) in $163 \mathrm{CRCHM}$ patients who received pre-operative chemotherapy using a Kaplan-Meier curve and univariate/multiple Cox model. Ten patients with rapidly progressing disease were further excluded in a sensitivity analysis. Seven risk factors (metachronous/synchronous metastases, differentiated grade of the primary tumor, number of metastases, size of the max metastasis, duration of pre-operative chemotherapy, radiologic response and pathologic regression) were used to stratify patients and investigate the beneficial features of adjuvant chemotherapy post-metastasectomy. The results indicated that adjuvant chemotherapy post-metastasectomy prolonged both RFS (median RFS: 3.3 vs. $10.2 \mathrm{~m}, \mathrm{P}=0.002$ ) and OS (median OS: 28.1 vs. $40.7 \mathrm{~m}, \mathrm{P}$ $=0.005$ ) in CRCHM patients who received pre-operative chemotherapy. After adjusting for other risk factors in a multiple Cox model, the adjuvant chemotherapy group was estimated to have a $54.0 \%$ relapse-free survival (hazard ratio $(H R)=0.46,95 \%$ confidence interval $(\mathrm{Cl}) 0.31-0.69, \mathrm{P}$ $<0.001$ ) and a $55.0 \%$ overall survival (HR [95 \% Cl]: 0.45 [0.26 - 0.78], $\mathrm{P}=0.005)$ advantage compared to patients without adjuvant chemotherapy. Additionally, the benefit of adjuvant chemotherapy post-liver resection remained in sensitivity analysis. After the risk stratification, patients with synchronous metastases, poor differentiation, $\geq 3$ metastases per patient, size of the maximum metastasis $>3 \mathrm{~cm}$, a short duration of pre-operative chemotherapy, radiologic response and poor pathologic regression seem to benefit more from adjuvant chemotherapy. To sum up, adjuvant chemotherapy post-metastasectomy might be considered for CRCHM patients who have received preoperative chemotherapy, especially for those with high-risk factors.
\end{abstract}

Key words: Colorectal cancer; Liver metastases; Adjuvant chemotherapy. 


\section{Background}

Colorectal cancer (CRC) is the third most common malignancy worldwide, accounting for $8 \%$ of new cancer cases in both men and women [1]. Liver is the most frequent site of CRC metastases, with an incidence of $25 \%$ at the initial diagnosis and $40-45 \%$ after the primary resection within two years [2-4]. When the metastatic disease is isolated to the liver, surgery resection is recommended as the most effective therapy, and it has a 5-year survival rate of $25-50 \%$ [5-7]. However, only $15-20 \%$ of patients with colorectal cancer hepatic metastases (CRCHM) received radical surgery at the initial diagnosis [8]. Even after the liver surgery, approximately two thirds of the patients relapse within the first 18 months, with the most frequent recurrence in the residual liver [9-12].

Pre-operative chemotherapy has been shown not only to convert the initially unresectable disease to resectable but also to improve the complete resection rate, facilitate hepatectomy, test the chemoresponsiveness, and increase disease free survival for resectable CRCHM [13-15]. However, the regimen-specific hepatic toxicity induced by chemotherapy cannot be ignored, which not only increases the risk of peri-operative mortality but also prolongs post-operative recovery and impairs quality of life. Therefore, the current view is that the metastasectomy should follow the pre-operative chemotherapy by $2-3$ months or should occur immediately when the metastases become resectable. After a radical resection, a physician is always in a dilemma about whether to continue to give the patient adjuvant chemotherapy. To our knowledge, there were no reports about the value of adjuvant chemotherapy in patients who had received preoperative chemotherapy.

The present study aimed to investigate the role of adjuvant chemotherapy post-metastasectomy in patients who received preoperative chemotherapy and to explore the potential beneficial factors by stratifying patients on several risks of recurrence.

\section{Methods}

\section{Patients}

Consecutive metastatic colorectal cancer patients, who underwent liver metastasectomy, were recruited between June 2002 and December 2015, at Sun Yat-sen University Cancer Center, China. Eligibility criteria included (a) pathologically and radiology confirmed CRCHM, (b) underwent fluoropyrimidine-based preoperative chemotherapy, (c) followed by liver metastasis resection with curative intent, (d) without extrahepatic metastases, and (e) adequate clinicopathological information and tumor tissue for analysis. Exclusion criteria were (a) incomplete surgery (R2 resection) and (b) disease progression during the pre-operative chemotherapy and having a regimen alteration after the liver metastasis resection.

\section{Treatment protocol}

CRC patients with liver metastases received preoperative fluoropyrimidine-based chemotherapy as determined by the physicians at Sun Yat-sen University Cancer Center. The fluoropyrimidinebased regimens included CAPEOX (oxaliplatin and capecitabine, 3-week intervals), XELIRI (irinotecan and capecitabine, 3-week intervals), FOLFOX (oxaliplatin, fluorouracil [FU] and Leucovorin [LV], 2-week intervals), FOLFIRI (irinotecan, FU and LV, 2-week intervals), FOLFOXIRI (oxaliplatin, irinotecan, FU and LV, 2-week intervals), capecitabine single agents (2-week intervals), FU/LV (2-week intervals) and the regimens above combined with hepatic arterial infusion (HAI) or bevacizumab or cetuximab. Bevacizumab was interrupted before and after the surgery at 6 weeks, and cetuximab was restrictively administered in patients with a wild-type KRAS exon 2. All surgeries were performed after the last preoperative chemotherapy administration, with a maximum delay of 1.5 months. For patients who underwent adjuvant chemotherapy after the liver resection, the regimen was the same as the preoperative chemotherapy. The eligible population was grouped into two categories (chemotherapy or surveillance arm) according to the treatment with or without adjuvant chemotherapy following the liver resection.

\section{Information collection and follow-up}

The clinicopathological data were collected from patient charts, which included demographic details, primary location, serum carcino-embyonic antigen (CEA) levels during the treatment, maximum size and number of liver metastases, regimens and cycles of pre-operative chemotherapy and pathological information of the primary tumor. Tumor stage was reclassified according to the Union International Control Cancer staging system, version 7 . Computerized tomography (CT) or a magnetic resonance imaging (MRI) procedure was used for radiological response assessment according to response evaluation criteria in solid tumors version 1.1 [16]. The initial images before the preoperative chemotherapy were compared with those after the end of the treatment but before the liver resection. The 
initial serum CEA levels were also compared with those after the preoperative chemotherapy but before surgery.

Pathological evaluation was performed on hematoxylin-eosin stained slides from formalin-fixed, paraffin-embedded $0.5 \mathrm{~mm}$ sections of hepatectomy specimens. All specimens were scored by two independent pathologists who were blinded to the patient's information, according to the tumor regression grades (TRG) criteria proposed by Rubbia-Brandt L [17]. There were five grades based on the percentage of residual tumor cells and the fibrosis in the TRG score system. Namely, TRG 1: an absence of residual cancer and an abundance of fibrosis; TRG 2: a large amount of fibrosis and scattered rare residual cancer cells; TRG 3: more residual cancer cells, but fibrosis predominates; TRG 4: presence of abundant residual cancer cells predominates over fibrosis; TRG 5: almost exclusively cancer with an absence of fibrosis. For patients who had more than one metastatic nodule, the worst pathological regressions (highest TRG) of all of the nodules per patient were used as the patient's pathological regression. Furthermore, a cut-off of 3 was used to classify the five TRG scores into two categories according to Carrasco J et al. [18]. Namely, TRG scores of 1-3 were defined as pathological regression, and TRG scores of 4-5 were defined as no pathological regression.

After the liver resection, follow-up was routinely performed every 3 months for the first 3 years and then every 6 months for 5 years, which included physical examination, CEA and CA19-9 levels, and a CT or MRI. The date of relapse and death were confirmed by the hospital records or phone contact with the patient or their relatives. This study was approved by the Ethical Board of Sun Yat-sen University Cancer Center and was conducted in accordance with the Helsinki Declaration of the World Medical Association. Given the non-interventional retrospective design, informed consent was not required in this study.

\section{Statistical analysis}

The role of adjuvant chemotherapy after the liver resection was tested for relapse-free survival (RFS) and overall survival (OS) using the Kaplan-Meier method with a log-rank test. RFS was defined as the time from liver surgery to the first relapse at any site or death due to any cause. OS was calculated from the metastasectomy to death from any cause. The univariate and multiple analyses were performed using Cox proportional hazard models. Comparisons between groups were analyzed with Student's $t$, Parson's Chi-squared or Fisher's exact test when appropriate. All statistical tests were performed using IBM SPSS software (version 22) with a two-tailed test. The variables were considered statistically significant for $\mathrm{P}$ values $<0.05$.

\section{Results}

\section{Patient characteristics}

The present study consisted of 163 patients with CRCHM who underwent hepatic metastasectomy after preoperative chemotherapy (Figure 1). Baseline characteristics are listed in Table 1 . The current population consisted primarily of males $(63.2 \%)$. The median age at the time of hepatic resection was 49 years (range: 28 - 83). The primary tumor was predominantly identified as colon cancer $(65.6 \%)$. All patients received FU-based chemotherapy, including 61 patients who received combinations with $\mathrm{HAI}(\mathrm{n}=$ $15)$ or bevacizumab $(n=22)$ or cetuximab $(n=24)$. The median number of cycles was 4 (range: 2 -12), and 78 patients $(47.9 \%)$ underwent a short-duration preoperative treatment (namely, $\leq 4$ cycles for 2-week regimens, $\leq 3$ cycles for 3 -week regimens), whereas 85 $(52.1 \%)$ received a long-duration treatment. The clinical and pathologic characteristics were comparable between the adjuvant chemotherapy and surveillance arms, as listed in Table 1.

\section{Role of adjuvant chemotherapy for hepatic metastasectomy}

As of the date of the last follow-up visit (August 2016), there were 123 patients (75.5\%) who had relapsed (median follow-up: 37.3 months; range: 7.2 171.7 months), including 117 with liver recurrence (50/63, $79.4 \%$ in the surveillance arm; $67 / 100,67 \%$ in the treatment arm). The median RFS and OS of patients in the surveillance arm were significantly worse than those of the patients with adjuvant chemotherapy (median RFS [mRFS]: 3.3 vs. $10.2 \mathrm{~m}, \mathrm{P}=$ 0.002; median OS [mOS]: 28.1 vs.40.7 m, P = 0.005; Figure 2). After adjusting for other risk factors in the multiple Cox model, the adjuvant chemotherapy group was estimated to have $54.0 \%$ relapse-free survival (hazard ratio $(\mathrm{HR})=0.46,95 \%$ confidence interval (CI) $0.31-0.69, \mathrm{P}<0.001$ ) and $55.0 \%$ overall survival (HR [95 \% CI]: 0.45 [0.26 - 0.78], P = 0.005) advantage compared to patients in the surveillance arm (Table 2).

Further, to avoid the impact of a shortened RFS on the decision regarding adjuvant chemotherapy, sensitivity analysis was performed by removing 10 patients who had relapsed within 1 month after the hepatic resection. The multivariate Cox analysis confirmed the role of adjuvant chemotherapy in prolonging RFS and OS (HR [95 \% CI]: 0.54 [0.35 - 
0.83 ], $\mathrm{P}=0.005 ;$ and $0.48[0.27-0.85], \mathrm{P}=0.012$, respectively; Table S1).

Table 1. Baseline characteristics $(N=163)$

\begin{tabular}{|c|c|c|c|}
\hline & $\begin{array}{l}\text { Adjuvant } \\
\text { chemotherapy } \\
\text { group }\end{array}$ & $\begin{array}{l}\text { Surveillance } \\
\text { group }\end{array}$ & P-value \\
\hline Patients & $100(61.3)$ & $63(38.7)$ & \\
\hline Age (years) & $49(28-83)$ & $48(28-78)$ & \\
\hline$\leq 65$ & $87(87.0)$ & $54(85.7)$ & 0.815 \\
\hline$>65$ & $13(13.0)$ & $9(14.3)$ & \\
\hline \multicolumn{4}{|l|}{ Gender } \\
\hline Male & $63(63.0)$ & $40(63.5)$ & 0.949 \\
\hline Female & $37(37.0)$ & $23(36.5)$ & \\
\hline \multicolumn{4}{|l|}{ Primary tumour site } \\
\hline Colon & $66(66.0)$ & $41(65.1)$ & 0.904 \\
\hline Rectum & $34(34.0)$ & $22(34.9)$ & \\
\hline \multicolumn{4}{|l|}{ Primary tumour grade } \\
\hline $\mathrm{G} 1, \mathrm{G} 2$ & $72(72.0)$ & $49(77.8)$ & 0.411 \\
\hline G3 & $28(28.0)$ & $14(22.2)$ & \\
\hline \multicolumn{4}{|l|}{ Histological subtype } \\
\hline Non-mucinous & $89(89.0)$ & $59(93.7)$ & 0.317 \\
\hline Mucinous & $11(11.0)$ & $4(6.3)$ & \\
\hline \multicolumn{4}{|l|}{ Primary tumour T-stage } \\
\hline $\mathrm{T} 1-2$ & $6(6.0)$ & $8(12.7)$ & 0.137 \\
\hline T3-4 & $94(94.0)$ & $55(87.3)$ & \\
\hline \multicolumn{4}{|l|}{ Primary tumour N-stage } \\
\hline No & $48(48.0)$ & $33(52.4)$ & 0.586 \\
\hline $\mathrm{N} 1-2$ & $52(52.0)$ & $30(47.6)$ & \\
\hline \multicolumn{4}{|l|}{ Pre-operative CEA } \\
\hline$\leq 5 \mathrm{ng} / \mathrm{ml}$ & $38(38.0)$ & $28(44.4)$ & 0.414 \\
\hline$>5 \mathrm{ng} / \mathrm{ml}$ & $62(62.0)$ & $35(55.6)$ & \\
\hline \multicolumn{4}{|l|}{ Metastases presentation } \\
\hline Synchronous & $82(82.0)$ & $49(77.8)$ & 0.509 \\
\hline Metachronous & $18(18.0)$ & $14(22.2)$ & \\
\hline Number of metastases per patient & $3(1-26)$ & $3(1-32)$ & \\
\hline$<3$ & $41(41.0)$ & $28(44.4)$ & 0.665 \\
\hline$\geq 3$ & $59(59.0)$ & $35(55.6)$ & \\
\hline Size of the max metastases $(\mathrm{cm})$ & $3(0.5-11.8)$ & $3(0.5-10.7)$ & 0.613 \\
\hline$\leq 3$ & $58(58.0)$ & $34(54.0)$ & \\
\hline$>3$ & $42(42.0)$ & $29(46.0)$ & \\
\hline \multicolumn{4}{|l|}{ Resection status } \\
\hline R0 & $80(80.0)$ & $44(69.8)$ & 0.139 \\
\hline $\mathrm{R} 1$ & $20(20.0)$ & $19(30.2)$ & \\
\hline \multicolumn{4}{|l|}{$\begin{array}{l}\text { Duration of preoperative } \\
\text { chemotherapy* }\end{array}$} \\
\hline Short-duration & $53(53.0)$ & $25(39.7)$ & 0.097 \\
\hline Long-duration & $47(47.0)$ & $38(60.3)$ & \\
\hline Pre-operative chemotherapy & & & 0.761 \\
\hline CT only & $71(71.0)$ & $46(73.0)$ & \\
\hline $\mathrm{CT}+\mathrm{Bev}$ & $15(15.0)$ & $7(11.1)$ & \\
\hline $\mathrm{CT}+$ Cet & $14(14.0)$ & $10(15.9)$ & \\
\hline \multicolumn{4}{|l|}{ CRS } \\
\hline $0-2$ & $62(62.0)$ & $32(50.8)$ & 0.159 \\
\hline $3-5$ & $38(38.0)$ & $31(49.2)$ & \\
\hline \multicolumn{4}{|l|}{ Radiological response\& } \\
\hline PR & $46(46.9)$ & $26(44.1)$ & 0.727 \\
\hline SD & $52(53.1)$ & $33(55.9)$ & \\
\hline \multicolumn{4}{|l|}{ TRG } \\
\hline $1-3$ & $63(63.0)$ & $37(58.7)$ & 0.586 \\
\hline $4-5$ & $37(37.0)$ & $26(41.3)$ & \\
\hline
\end{tabular}

Data are median (rang) or number $(\%)$. $\mathrm{CEA}=$ cantigen antigen. $\mathrm{CT}=$ chemotherapy. Bev $=$ bevacizumab. Cet $=$ cetuximab. $C R S=$ clinical risk score $. P R=$ partial response. $\mathrm{SD}=$ stable disease. $T R G=$ tumor regression grade

*Short-duration of preoperative chemotherapy: $\leq 4$ cycles for 2 -weekly regimens, $\leq$ 3 cycles for 3-weekly regimens; long-duration of preoperative chemotherapy: $>4$ cycles for 2-weekly regimens, > 3 cycles for 3-weekly regimens. \&Six of 163 patients were unevaluable for radiological response, because of the initial computerised tomography or magnetic resonance imaging performed in other hospitals.

\section{Risk stratification}

To explore the potential beneficial factors for adjuvant chemotherapy, patients in the present study were further classified into two risk stratifications by metastases history (metachronous/synchronous metastases), differentiated grade of the primary tumor (G1-2/G3), number of metastases $(<3 / \geq 3$ per patient), size of the max metastasis $(\leq 3 />3 \mathrm{~cm})$, duration of pre-operative chemotherapy (short/long duration), radiologic response (partial response $[\mathrm{PR}] /$ stable disease [SD]), and pathologic regression (regression/no regression).

Adjuvant chemotherapy post-metastasectomy was clinically and statistically significantly beneficial for both RFS and OS in patients with synchronous metastases (mRFS 2.6 vs. $10.2 \mathrm{~m}, \mathrm{P}<0.001$; mOS $25.0 \mathrm{~m}$ vs. not reached, $\mathrm{P}<0.001$ ), pathologic grade $\mathrm{G} 3$ (mRFS 1.1 vs. $10.5 \mathrm{~m}, \mathrm{P}=0.003$; mOS $22.0 \mathrm{~m}$ vs. not reached, $\mathrm{P}$ $=0.007), \geq 3$ metastases per patient (mRFS 1.8 vs. 8.4 $\mathrm{m}, \mathrm{P}<0.001$; mOS 24.5 vs. $34.6 \mathrm{~m}, \mathrm{P}=0.028)$, the size of the maximum metastasis $>3 \mathrm{~cm}$ (mRFS 3.3 vs. 10.2 $\mathrm{m}, \mathrm{P}=0.006$; mOS 25.0 vs. $40.7 \mathrm{~m}, \mathrm{P}=0.001$ ) and a short duration of pre-operative chemotherapy (mRFS 4.6 vs. $10.2 \mathrm{~m}, \mathrm{P}=0.012$; $\operatorname{mOS} 25.0$ vs. $35.6 \mathrm{~m}, \mathrm{P}=$ 0.004), when compared with the surveillance arm (Table 3, Figure S1). Inversely, there was no statistically significant difference between the adjuvant chemotherapy arm and the surveillance arm in patients with metachronous metastases, Grade 1-2, $<3$ metastases per patient, the size of the max metastasis $\leq 3 \mathrm{~cm}$, and a long duration of preoperative chemotherapy. However, a beneficial trend was observed in the adjuvant chemotherapy arm in subgroups with grade $1-2,<3$ metastases per patient and a long duration preoperative treatment (Table 3, Figure S1).

For the risk stratification by tumor radiologic evaluation, patients who obtained PR for preoperative chemotherapy can benefit from adjuvant chemotherapy post-metastasectomy on RFS (mRFS 1.4 vs. $10.7 \mathrm{~m}, \mathrm{P}<0.001$ ), whereas for those with SD, the benefit trend of adjuvant chemotherapy did not reach statistical significance. However, both the patients with PR and SD obtained a longer overall survival from treatment with adjuvant chemotherapy compared to observation post-surgery (mOS $36.1 \mathrm{vs}$. not reached, $\mathrm{P}=0.049$; and 25.3 vs. $38.0 \mathrm{~m}, \mathrm{P}=0.012$, respectively; Table 3, Figure S1).

Interestingly, patients who obtained pathological regression (TRG1-3) from the preoperative chemotherapy seemed to benefit little from the continued adjuvant chemotherapy post-metastasectomy (surveillance arm vs. adjuvant chemotherapy arm: mRFS 5.2 vs.10.5 months, $\mathrm{P}=$ 0.172; mOS 48.0 vs. 40.7 months, $\mathrm{P}=0.779$, 
respectively), whereas for patients who did not obtain pathological regression from prior chemotherapy (TRG 4-5), adjuvant chemotherapy exhibited an obvious survival benefit compared with surveillance only (mRFS 1.6 vs. 8.7 months, $\mathrm{P}=0.001$; mOS 20.1 vs. 43.1 months, $\mathrm{P}<0.001$ ).

\section{Discussion}

In the present study, 163 CRCHM patients with liver resection after pre-operative chemotherapy were retrospectively analyzed. The results showed a benefit in both RFS and OS in the adjuvant chemotherapy arm compared to the observation arm. Considering the impact of aggressive disease on post-surgery decisions, we further excluded 10 patients who had rapidly progressing disease within 1 month in the sensitivity analysis. The adjuvant chemotherapy remained to prolong the RFS and OS for patients after liver resection. Although the study is limited by its retrospective design, to our knowledge, it is the first report to focus on the necessity of continued chemotherapy post-metastasectomy after prior pre-operative chemotherapy.

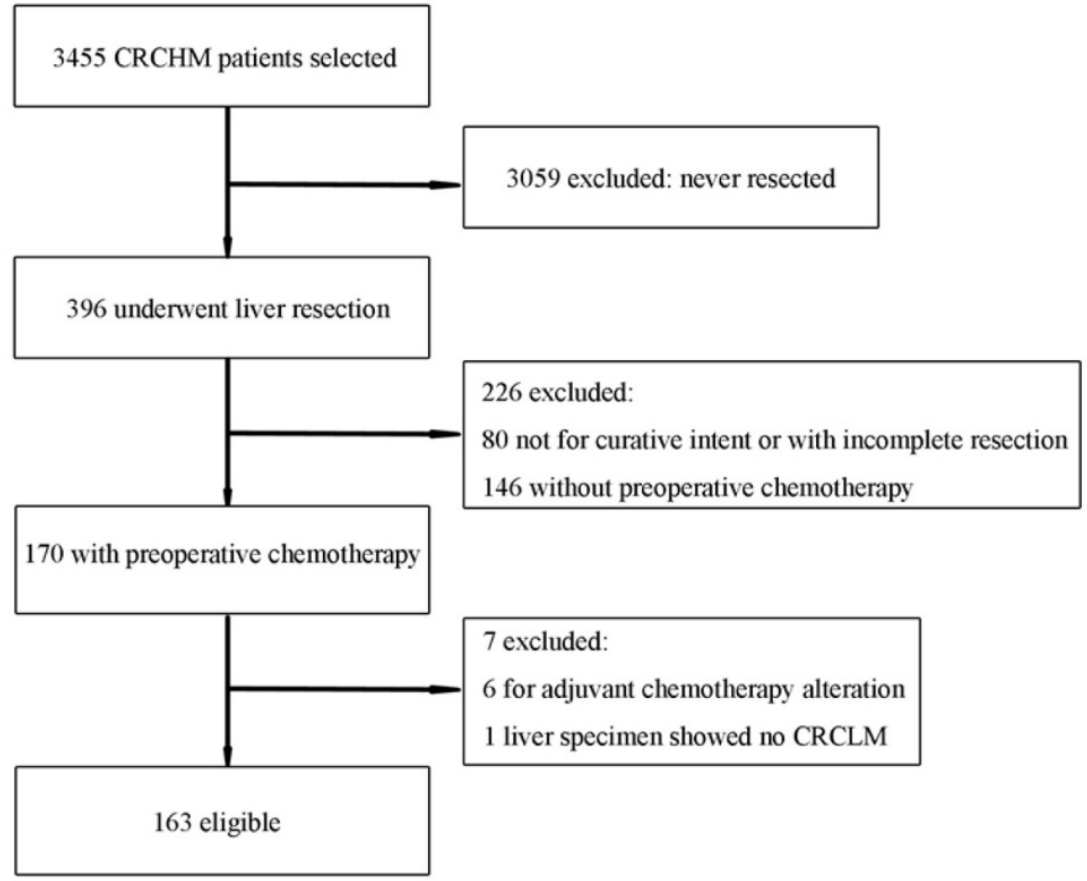

Figure 1. Flow chart of patient inclusion
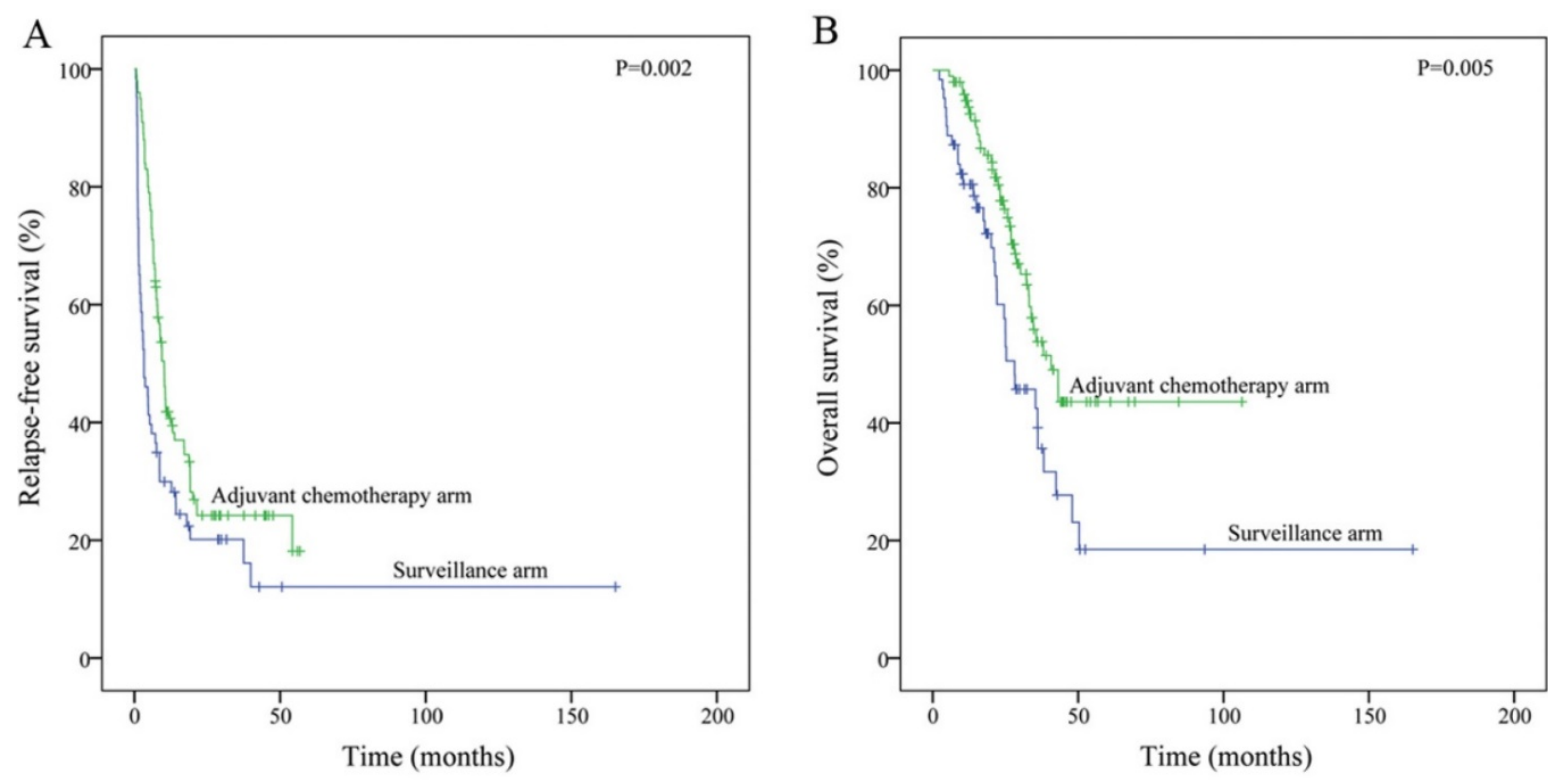

Figure 2. Kaplan-Meier curves of Relapse-free survival (A) and Overall survival (B) according to treatment post-liver resection, based on the whole population ( $N$ $=163$ ). 
Table 2. Predictive factors for survival by univariate and multivariate analysis based on the whole population $(\mathrm{N}=163)$

\begin{tabular}{|c|c|c|c|c|c|c|c|c|c|}
\hline & & \multicolumn{4}{|c|}{ Relapse-free survival } & \multicolumn{4}{|l|}{ Overall survival } \\
\hline & & \multicolumn{2}{|c|}{ Univariate analysis } & \multicolumn{2}{|c|}{ Multivariate analysis } & \multicolumn{2}{|c|}{ Univariate analysis } & \multicolumn{2}{|c|}{ Multivariate analysis } \\
\hline & & HR (95\%CI) & P-value & HR $(95 \% \mathrm{CI})$ & $\begin{array}{l}\text { P-val } \\
\text { ue }\end{array}$ & $\mathrm{HR}(95 \% \mathrm{CI})$ & P-value & HR $(95 \% \mathrm{CI})$ & P-value \\
\hline Age & $>65$ vs. $\leq 65$ & $1.09(0.65-1.82)$ & 0.744 & $1.24(0.67-2.20)$ & 0.469 & $1.37(0.70-2.67)$ & 0.363 & $2.03(0.95-4.34)$ & 0.066 \\
\hline Gender & male vs. female & $1.04(0.72-1.49)$ & 0.850 & $1.08(0.71-1.65)$ & 0.716 & $0.80(0.49-1.28)$ & 0.345 & $0.75(0.43-1.30)$ & 0.308 \\
\hline Primary tumour & rectal vs. colon & $1.21(0.83-1.73)$ & 0.317 & $1.11(0.72-1.72)$ & 0.635 & $1.13(0.70-1.82)$ & 0.621 & $1.19(0.67-2.09)$ & 0.552 \\
\hline Tumor grade & G3 vs. G1-2 & $0.96(0.79-1.18)$ & 0.709 & $1.06(0.85-1.33)$ & 0.615 & $0.84(0.63-1.12)$ & 0.244 & $0.91(0.66-1.25)$ & 0.542 \\
\hline T-stage & T3-4 vs. T1-2 & $0.90(0.47-1.72)$ & 0.746 & $1.13(0.56-2.29)$ & 0.740 & $1.17(0.47-2.90)$ & 0.738 & $1.15(0.41-3.21)$ & 0.786 \\
\hline N-stage & N1-2 vs. N0 & $1.33(0.93-1.89)$ & 0.121 & $1.38(0.92-2.06)$ & 0.123 & $1.22(0.76-1.94)$ & 0.413 & $1.46(0.83-2.57)$ & 0.186 \\
\hline Pre-operative CEA & $>5 \mathrm{ng} / \mathrm{ml} v s . \leq 5 \mathrm{ng} / \mathrm{ml}$ & $1.10(0.76-1.58)$ & 0.619 & $.89(0.59-1.34)$ & 0.567 & $1.67(1.01-2.74)$ & 0.045 & $1.80(1.03-3.16)$ & 0.039 \\
\hline Metastases presentation & $\begin{array}{l}\text { metachronous } v s . \\
\text { synchronous }\end{array}$ & $1.03(0.67-1.60)$ & 0.882 & $.95(0.59-1.55)$ & 0.848 & $1.07(0.62-1.88)$ & 0.802 & $0.80(0.42-1.54)$ & 0.508 \\
\hline Number of metastases & $\geq 3$ vs. $<3$ & $1.54(1.07-2.21)$ & 0.020 & $1.42(0.93-2.16)$ & 0.108 & $1.42(0.87-2.31)$ & 0.159 & $1.41(0.78-2.54)$ & 0.251 \\
\hline $\begin{array}{l}\text { Size of the max metastases } \\
(\mathrm{cm})\end{array}$ & $>3$ vs. $\leq 3$ & $1.13(0.79-1.61)$ & 0.511 & $1.09(0.72-1.64)$ & 0.687 & $1.26(0.79-2.00)$ & 0.339 & $0.95(0.53-1.71)$ & 0.861 \\
\hline Resection status & R1-2 vs. R0 & $2.79(1.87-4.16)$ & $<0.001$ & $2.10(1.34-3.31)$ & 0.001 & $2.57(1.60-4.14)$ & $<0.001$ & $2.07(1.15-3.73)$ & 0.016 \\
\hline $\begin{array}{l}\text { Duration of preoperative } \\
\mathrm{CT}\end{array}$ & long vs. short & $1.10(0.77-1.57)$ & 0.590 & $1.08(0.72-1.62)$ & 0.721 & $0.91(0.57-1.45)$ & 0.685 & $0.72(0.41-1.26)$ & 0.247 \\
\hline \multirow[t]{2}{*}{ Chemotherapy backbone } & $\begin{array}{l}\text { CT with Bev vs. CT } \\
\text { only }\end{array}$ & $1.06(0.63-1.79)$ & 0.815 & $1.30(0.74-2.29)$ & 0.366 & $1.09(0.55-2.16)$ & 0.800 & $1.51(0.70-3.26)$ & 0.289 \\
\hline & CT with Cet vs. CT only & $1.42(0.88-2.28)$ & 0.156 & $1.07(0.62-1.87)$ & 0.804 & $1.31(0.72-2.38)$ & 0.381 & $1.66(0.83-3.29)$ & 0.150 \\
\hline Response Evaluation\& & SD vs. PR & $0.85(0.59-1.22)$ & 0.386 & $.78(0.50-1.20)$ & 0.254 & $1.23(0.76-2.01)$ & 0.397 & $1.57(0.85-2.90)$ & 0.152 \\
\hline TRG & TRG4-5 vs. TRG1-3 & $1.67(1.17-2.38)$ & 0.005 & $1.57(1.04-2.38)$ & 0.033 & $1.69(1.06-2.70)$ & 0.027 & $1.59(0.92-2.76$ & 0.097 \\
\hline $\begin{array}{l}\text { Postoperative } \\
\text { chemotherapy }\end{array}$ & yes $v s$. no & $0.58(0.40-0.83)$ & 0.003 & $0.46(0.31-0.69)$ & $\begin{array}{l}< \\
0.001\end{array}$ & $0.52(0.33-0.83)$ & 0.006 & $0.45(0.26-0.78)$ & 0.005 \\
\hline
\end{tabular}

$\mathrm{CEA}=$ cantigen $. \mathrm{CT}=$ chemotherapy. Bev $=$ bevacizumab. Cet $=$ cetuximab. $\mathrm{CRS}=$ clinical risk score. $\mathrm{PR}=$ partial response. $\mathrm{SD}=$ stable disease. $\mathrm{TRG}=$ tumor regression grade. \&Six of 163 patients were unevaluable for radiological response.

Table 3. Role of adjuvant chemotherapy post-metastasectomy after the risk stratification

\begin{tabular}{|c|c|c|c|c|c|c|}
\hline \multirow[t]{2}{*}{ Risk stratification } & \multicolumn{3}{|c|}{ median RFS (months) } & \multicolumn{3}{|c|}{ median OS (months) } \\
\hline & Surveillance & Adjuvant CT & P-value & Surveillance & Adjuvant CT & P-value \\
\hline \multicolumn{7}{|l|}{ Metastases history } \\
\hline synchronous $(\mathrm{n}=131)$ & 2.6 & 10.2 & $<0.001$ & 25.0 & NR & $<0.001$ \\
\hline metachronous $(n=32)$ & 5.2 & 7.5 & 0.344 & 48.0 & 33.0 & 0.250 \\
\hline \multicolumn{7}{|l|}{ Differentiated grade } \\
\hline G1-2 (n=121) & 3.3 & 9.4 & 0.062 & 28.1 & 34.6 & 0.118 \\
\hline $\mathrm{G} 3(\mathrm{n}=42)$ & 1.1 & 10.5 & 0.003 & 22.0 & NR & 0.007 \\
\hline \multicolumn{7}{|l|}{ Number of metastases } \\
\hline$<3$ per pateint $(n=69)$ & 8.6 & 11.8 & 0.478 & 28.1 & NR & 0.131 \\
\hline$\geq 3$ per pateint $(n=94)$ & 1.8 & 8.4 & $<0.001$ & 24.5 & 34.6 & 0.028 \\
\hline \multicolumn{7}{|l|}{ Size of the max metastasis } \\
\hline$\leq 3 \mathrm{~cm}(\mathrm{n}=92)$ & 3.3 & 9.4 & 0.114 & 38.1 & 34.6 & 0.315 \\
\hline$>3 \mathrm{~cm}(\mathrm{n}=71)$ & 3.3 & 10.2 & 0.006 & 25.0 & 40.7 & 0.001 \\
\hline \multicolumn{7}{|c|}{ Duration of pre-operative chemotherapy } \\
\hline short-duration $(\mathrm{n}=78)$ & 4.6 & 10.2 & 0.012 & 25.0 & 35.6 & 0.004 \\
\hline long duration $(\mathrm{n}=85)$ & 3 & 10.2 & 0.073 & 35.4 & 40.7 & 0.172 \\
\hline \multicolumn{7}{|l|}{ Radiologic response\& } \\
\hline$P R(n=72)$ & 1.4 & 10.7 & $<0.001$ & 36.1 & NR & 0.049 \\
\hline $\mathrm{SD}(\mathrm{n}=85)$ & 5.9 & 8.4 & 0.554 & 25.3 & 38.0 & 0.012 \\
\hline \multicolumn{7}{|l|}{ Pathologic regression } \\
\hline TRG $1-3(n=100)$ & 5.2 & 10.5 & 0.172 & 48.0 & 40.7 & 0.779 \\
\hline TRG4-5 $(n=63)$ & 1.6 & 8.7 & 0.001 & 20.1 & 43.1 & $<0.001$ \\
\hline
\end{tabular}

$\mathrm{CT}=$ chemotherapy. $\mathrm{PR}=$ partial response $\mathrm{SD}=$ stable disease. $\mathrm{TRG}=$ tumor regression grade. $\mathrm{NR}=$ not reached. \&Six patients were unevaluable for radiological response.

Chemotherapy, as an approach that can potentially improve survival, is proposed for peri-operative treatment, although the optimal regimens and duration have not been defined. The EORTC40983 trial randomly assigned 364 patients with resectable mCRC to 6 months of peri-operative mFOLFOX4 chemotherapy ( 3 months before and 3 months after) or surgery followed by observation. A 9.2\% increase in PFS was observed in patients undergoing resection with peri-operative chemotherapy compared to surgery alone (HR [95\% CI] $0.73[0.55-0.97], P=0$.025) [19]. However, the difference in OS between the groups did not reach statistical significance after the long-term follow-up [20]. This was the first trial to confirm the role of perioperative chemotherapy for liver resection with adequate statistical power. However, no further analysis was performed on the value of 3 months of 
post-surgery chemotherapy on the premise of pre-operative chemotherapy. Other randomized trials were performed to compare adjuvant systemic chemotherapy following metastasectomy with surgery alone [21-24]. The results were inconsistent with inadequate power. Mitry et al then pooled the 278 patients from the EORTC 40923 trial and FFCD 9002 trial and reported that adjuvant FU/LV chemotherapy improved the PFS (HR [95 \% CI] 1.32 [1.00 - 1.76], $\mathrm{P}=0.058)$ and OS (HR [95 \% CI] 1.32 [0.95 - 1.82], $\mathrm{P}=0.095$ ) of patients with liver resection with critical statistical significance [25]. Nevertheless, none of these studies enrolled patients who had received prior chemotherapy before liver resection.

In a further stratification analysis of the present study, FU-based adjuvant chemotherapy dramatically improved the RFS and OS in the synchronous subgroup but not in the metachronous groups. This finding was consistent with previous analyses, conducted by Wang et al, who showed that synchronous metastatic CRC seemed to have a greater survival benefit from the adjuvant chemotherapy post-liver resection, although the study didn't restrict the included patients with having pre-operative chemotherapy [26]. This fact was most likely due to the poor prognosis of patients with synchronous metastases, who need a more aggressive post-surgery treatment, such as chemotherapy, to treat the micro-metastatic disease. Alternatively, most patients with metachronous metastases had a history of prior adjuvant chemotherapy post-primary tumor resection, which most likely reduced the sensitivity to re-chemotherapy. In addition, our results showed that a patient with risk factors, including more liver metastases, larger sized metastases or a poorer degree of tumor differentiation, which implies a more malignant disease, can have a greater survival advantage from post-metastasectomy chemotherapy.

Patients who had obtained a partial response from the pre-operative chemotherapy can benefit more from continuous post-liver resection chemotherapy. These patients represented the population with higher chemo-responsiveness, whereas adjuvant chemotherapy can prolong the overall survival, even for those whose best response was only stable disease.

In the present study, patients who did not obtain a satisfactory pathological regression (TRG 4-5) from pre-operative chemotherapy can benefit more from continuous post-surgery chemotherapy, but those with a satisfactory pathological regression (TRG 1-3) benefit little from adjuvant chemotherapy. This result was similar to the phenomenon observed in locally advanced rectal cancer, where adjuvant chemotherapy was not necessary for patients with a complete pathological response after neoadjuvant chemoradiotherapy [27]. The comparatively better biological characteristics may weaken the role of adjuvant chemotherapy. Instead, patients characterized by poor biological disease, such as TRG 4-5 from prior treatment, need more aggressive adjuvant chemotherapy. In another study from South Korea (ADORE study), 321 rectal cancer patients who underwent preoperative chemoradiotherapy and total mesorectal excision with post-operative stage II or III were randomized into an adjuvant FOLFOX chemotherapy group or 5-Fu/LV group. The population enrolled excluded the patients with a complete pathological response. The results demonstrated the benefit of adding oxaliplatin to fluoropyrimidine-based adjuvant therapy in this special population [28]. Alternatively, the degree of pathological regression was associated with the duration of chemotherapy [29]. It was consistent with the results that patients with short-duration pre-operative chemotherapy may benefit more from the adjuvant chemotherapy compared to those with long-duration treatment, because they may be characterized with poorer pathological regression.

Although the two arms (surveillance/adjuvant chemotherapy arm) were balanced among the risk factors, the retrospective design and small sample size weaken the power of the conclusion in our study, particularly for the subgroup analysis, which was based on a smaller sample size. The preliminary results should be interpreted with caution.

\section{Conclusions}

Adjuvant chemotherapy post-hepa-metastasectomy can be considered even for those who had received pre-operative chemotherapy, and the management plan should be determined based on the risk factors for each patient. Further prospective clinical trials are warranted.

\section{Supplementary Material}

Supplementary figures and tables.

http://www.jcancer.org/v08p1179s1.pdf

\section{Abbreviations}

CRCHM: Colorectal cancer hepatic metastases; RFS: Relapse-free survival; OS: Overall survival; CRC: Colorectal cancer; FU: fluorouracil; LV: Leucovorin; HAI: Hepatic arterial infusion; CEA: Carcino-embyonic antigen; TRG: Tumor regression grades.

\section{Acknowledgments}

Our gratitude is expressed to all the patients for the participation in this study. We also appreciate all 
colleagues in Sun Yat-sen University Cancer Center, who have participated in performing the treatment in the current study. Medbanks (Beijing) Network Technology Co., Ltd was thanked for data collection.

\section{Ethics Committee Approval and Patient Consent}

Ethical approval and consent to participate: All procedures performed in studies involving human participants were in accordance with the ethical standards of the institutional and/or national research committee and with the 1964 Helsinki declaration and its later amendments or comparable ethical standards. A waiver of informed consent was request, and approval was obtained from independent ethics committees of Sun Yat-sen University Cancer Center.

\section{Competing Interests}

The authors have declared that no competing interest exists.

\section{References}

1. Siegel RL, Miller KD, Jemal A. Cancer statistics, 2016. CA Cancer J Clin 2016; 66: 7-30

2. Tomlinson JS, Jarnagin WR, DeMatteo RP, et al. Actual 10-year survival after resection of colorectal liver metastases defines cure. J Clin Oncol 2007; 25: $4575-80$

3. Van Cutsem E, Cervantes A, Nordlinger B, et al. Metastatic colorectal cancer: ESMO Clinical Practice Guidelines for diagnosis, treatment and follow-up. Ann Oncol 2014; 25 Suppl 3: iii1-9

4. Rees M, Tekkis PP, Welsh FK, et al. Evaluation of long-term survival after hepatic resection for metastatic colorectal cancer: a multifactorial model of 929 patients. Ann Surg 2008; 247: 125-35

5. Biasco G, Derenzini E, Grazi G, et al. Treatment of hepatic metastases from colorectal cancer: many doubts, some certainties. Cancer Treat Rev 2006; 32: 214-28

6. Abdalla EK, Vauthey JN, Ellis LM, et al. Recurrence and outcomes following hepatic resection, radiofrequency ablation, and combined resection/ablation for colorectal liver metastases. Ann Surg 2004; 239: 818-25; discussion 825-7

7. Parks R, Gonen M, Kemeny N, et al. Adjuvant chemotherapy improves survival after resection of hepatic colorectal metastases: analysis of data from two continents. J Am Coll Surg 2007; 204: 753-61; discussion 761-3

8. Kopetz S, Chang GJ, Overman MJ, et al. Improved survival in metastatic colorectal cancer is associated with adoption of hepatic resection and improved chemotherapy. J Clin Oncol 2009; 27: 3677-83

9. Fong Y. Hepatic colorectal metastasis: current surgical therapy, selection criteria for hepatectomy, and role for adjuvant therapy. Adv Surg 2000; 34: 351-81

10. de Jong MC, Pulitano C, Ribero D, et al. Rates and patterns of recurrence following curative intent surgery for colorectal liver metastasis: an international multi-institutional analysis of 1669 patients. Ann Surg 2009; 250: $440-8$

11. Penna C, Nordlinger B. Colorectal metastasis (liver and lung). Surg Clin North Am 2002; 82: 1075-90, x-xi

12. Brandi G, De Lorenzo S, Nannini M, et al. Adjuvant chemotherapy for resected colorectal cancer metastases: Literature review and meta-analysis. World J Gastroenterol 2016; 22: 519-33

13. Power DG, Kemeny NE. Role of adjuvant therapy after resection of colorectal cancer liver metastases. J Clin Oncol 2010; 28: 2300-9

14. Kemeny N. Presurgical chemotherapy in patients being considered for liver resection. Oncologist 2007; 12: 825-39

15. Adam R, Delvart V, Pascal G, et al. Rescue surgery for unresectable colorectal liver metastases downstaged by chemotherapy: a model to predict long-term survival. Ann Surg 2004; 240: 644-57; discussion 657-8

16. Eisenhauer EA, Therasse P, Bogaerts J, et al. New response evaluation criteria in solid tumours: revised RECIST guideline (version 1.1). Eur J Cancer 2009; 45: 228-47

17. Rubbia-Brandt L, Giostra E, Brezault C, et al. Importance of histological tumor response assessment in predicting the outcome in patients with colorectal liver metastases treated with neo-adjuvant chemotherapy followed by liver surgery. Ann Oncol 2007; 18: 299-304
18. Carrasco J, Gizzi M, Pairet $G$, et al. Pathological responses after angiogenesis or EGFR inhibitors in metastatic colorectal cancer depend on the chemotherapy backbone. Br J Cancer 2015; 113: 1298-304

19. Nordlinger B, Sorbye H, Glimelius B, et al. Perioperative chemotherapy with FOLFOX4 and surgery versus surgery alone for resectable liver metastases from colorectal cancer (EORTC Intergroup trial 40983): a randomised controlled trial. Lancet 2008; 371: 1007-16

20. Nordlinger B, Sorbye H, Glimelius B, et al. Perioperative FOLFOX4 chemotherapy and surgery versus surgery alone for resectable liver metastases from colorectal cancer (EORTC 40983): long-term results of a randomised, controlled, phase 3 trial. Lancet Oncol 2013; 14: 1208-15

21. Lopez-Ladron A Salvador J, Bernabr R, et al. Observation versus postoperative chemotherapy after resection of liver metastases in patients with advanced colorectal cancer. Proc Amer Soc Clin Oncol. 2003; 22: 373(suppl): abstract1497.

22. Langer B, Bleiberg H, Labianca R, et al. Fluorouracil (FU) plus l-leucovorin (l-LV) versus observation after potentially curative resection of liver or lung metastases from colorectal cancer (CRC): results of the ENG (EORTC/NCIC CTG/GIVIO) randomized trial. Proc Amer Soc Clin Oncol. 2002; 21: 149a(suppl): abstract592.

23. O'Connell MJ, Adson MA, Schutt AJ, et al. Clinical trial of adjuvant chemotherapy after surgical resection of colorectal cancer metastatic to the liver. Mayo Clin Proc 1985; 60: 517-20

24. Portier G, Elias D, Bouche O, et al. Multicenter randomized trial of adjuvant fluorouracil and folinic acid compared with surgery alone after resection of colorectal liver metastases: FFCD ACHBTH AURC 9002 trial. J Clin Oncol 2006; 24: 4976-82

25. Mitry E, Fields AL, Bleiberg H, et al. Adjuvant chemotherapy after potentially curative resection of metastases from colorectal cancer: a pooled analysis of two randomized trials. J Clin Oncol 2008; 26: 4906-11

26. Wang X, Hershman DL, Abrams JA, et al. Predictors of survival after hepatic resection among patients with colorectal liver metastasis. Br J Cancer 2007; 97: 1606-12

27. Zhou J, Qiu H, Lin G, et al. Is adjuvant chemotherapy necessary for patients with pathological complete response after neoadjuvant chemoradiotherapy and radical surgery in locally advanced rectal cancer? Long-term analysis of 40 ypCR patients at a single center. Int J Colorectal Dis 2016; 31: 1163-8

28. Hong YS, Nam BH, Kim KP, et al. Oxaliplatin, fluorouracil, and leucovorin versus fluorouracil and leucovorin as adjuvant chemotherapy for locally advanced rectal cancer after preoperative chemoradiotherapy (ADORE): an open-label, multicentre, phase 2, randomised controlled trial. Lancet Oncol 2014; 15: 1245-53

29. Sule EA, Nzegwu MA. Attaining pathological complete regression for breast conservation - A pilot experience in a developing country. Ann Med Surg (Lond) 2016; 9: 61-6 